\title{
Multifocal motor neuropathy: controversies and priorities
}

\author{
Wei Zhen Yeh (D) , 1,2 P James Dyck, ${ }^{3}$ Leonard H van den Berg, ${ }^{4}$ Matthew C Kiernan, ${ }^{5,6}$ \\ Bruce V Taylor ${ }^{1,7}$
}

'Department of Neurology, Royal Hobart Hospital, Hobart, Tasmania, Australia ${ }^{2}$ Department of Neuroscience, Central Clinical School, Monash University, Melbourne, Victoria, Australia

${ }^{3}$ Department of Neurology, Mayo Clinic, Rochester, Minnesota, USA

${ }^{4} U M C$ Utrecht Brain Center, Department of Neurology, University Medical Center Utrecht, Utrecht, Netherlands ${ }^{5}$ Bushell Chair of Neurology, Brain and Mind Centre, University of Sydney, Sydney, New South Wales, Australia ${ }^{6}$ Department of Neurology, Royal Prince Alfred Hospital, Camperdown, New South Wales, Australia

${ }^{7}$ Menzies Institute for Medical Research, University of Tasmania, Hobart, Tasmania, Australia

\section{Correspondence to} Professor Bruce V Taylor, Menzies Institute for Medica Research, University of Tasmania, Hobart, Tasmania, Australia; bruce.taylor@utas. edu.au

Received 1 July 2019 Revised 14 August 2019 Accepted 31 August 2019 Published Online First 11 September 2019

Check for updates

(C) Author(s) (or their employer(s)) 2020. No commercial re-use. See rights and permissions. Published by BMJ.

To cite: Yeh WZ, Dyck PJ, van den Berg LH, et al. J Neurol Neurosurg Psychiatry

2020:91:140-148.

\section{ABSTRACT}

Despite 30 years of research there are still significant unknowns and controversies associated with multifocal motor neuropathy (MMN) including disease pathophysiology, diagnostic criteria and treatment. Foremost relates to the underlying pathophysiology, specifically whether MMN represents an axonal or demyelinating neuropathy and whether the underlying pathophysiology is focused at the node of Ranvier. In turn, this discussion promotes consideration of therapeutic approaches, an issue that becomes more directed in this evolving era of precision medicine. It is generally accepted that MMN represents a chronic progressive immune-mediated motor neuropathy clinically characterised by progressive asymmetric weakness and electrophysiologically by partial motor conduction block. Anti-GM1 IgM antibodies are identified in at least $40 \%$ of patients. There have been recent developments in the use of neuromuscular ultrasound and MRI to aid in diagnosing MMN and in further elucidation of its pathophysiological mechanisms. The present Review will critically analyse the knowledge accumulated about MMN over the past 30 years, culminating in a state-of-the-art approach to therapy.

\section{INTRODUCTION}

Despite 30 years of research there are still significant unknowns and controversies associated with multifocal motor neuropathy (MMN) including disease pathophysiology, diagnostic criteria and treatment (table 1). Foremost relates to the underlying pathophysiology, specifically whether MMN represents an axonal or demyelinating neuropathy and whether the underlying pathophysiology is focused at the node of Ranvier. In turn, this discussion promotes consideration of therapeutic approaches, an issue that becomes more directed in this evolving era of precision medicine. In terms of knowns, it is generally accepted that $\mathrm{MMN}$ represents a chronic progressive immune-mediated motor neuropathy, clinically characterised by progressive asymmetric weakness and electrophysiologically by partial motor conduction block. While MMN may be considered rare with prevalence estimated at 0.6 to 2 per 100000 population, ${ }^{12}$ it is likely to be an under-recognised entity. In addition to improved understanding of the basic mechanisms there have also been recent developments in the use of neuromuscular ultrasound and MRI to aid in diagnosing MMN and in further elucidation of its pathophysiological mechanisms. As such, the present Review will critically analyse the knowledge accumulated about $\mathrm{MMN}$ over the past 30 years, culminating in a state-of-the-art approach to therapy.

\section{CLINICAL PHENOTYPE}

Classically MMN presents as an asymmetrical upper limb pure motor multiple mononeuropathy, with often prominent wasting despite a short history of weakness. MMN more commonly affects males, with a male-to-female ratio of 2.7:1. Age at symptom onset can range between 20 and 70 years with a mean age of 40 years. ${ }^{134}$ The first symptom is most commonly distal upper limb weakness but with relative sparing of finger flexors. ${ }^{1}$ Foot drop is the first symptom in a third of patients. Many with initial lower limb involvement later develop upper limb symptoms. Involvement of the non-dominant upper limb is more frequently reported. Fasciculations and cramps are prominent and observed in up to $40 \%$ of patients, and in some cases may generate local muscle hypertrophy. Aggravation of weakness by cold is common and reported in $83 \%$ of cases in one study. ${ }^{5}$ Deep tendon reflexes are usually reduced in the affected limb, but may also be normal.

The presence of sensory symptoms, signs or neurophysiological abnormalities particularly at diagnosis or early in disease should raise significant diagnostic uncertainty and prompt further investigation including consideration of targeted nerve biopsy. However, in one case series, $22 \%$ had abnormal vibration sense in the distal lower limb, with these cases having had a longer median disease duration compared with those without sensory findings. ${ }^{1}$

Involvement beyond the limbs is rare, with scattered reports of phrenic and cranial nerve involvement. ${ }^{6}$ Diagnostic criteria for $\mathrm{MMN}$ were proposed by the European Federation of Neurological Societies and the Peripheral Nerve Society and published in 2010 (box 1 lists the clinical criteria for MMN). ${ }^{7}$

\section{Clinical neurophysiology}

Identification of motor conduction block (CB) is the key neurophysiological criterion in the diagnosis of MMN (box 2). ${ }^{7}$ The two most commonly affected nerves are the median and ulnar nerves, ${ }^{3}$ in their forearm segments and not at typical compression sites. The $\mathrm{CB}$ observed in $\mathrm{MMN}$ is unique in that it affects motor fibres exclusively with normal sensory conduction through the same segment in mixed nerves. ${ }^{23}$ Additionally the block is focal and 
Table 1 Controversies and priorities in multifocal motor neuropathy (MMN)

\begin{tabular}{|c|c|}
\hline Diagnosis & $\begin{array}{l}\text { What is the role for nerve MRI and } \\
\text { ultrasound? } \\
\text { Can a patient have MMN despite } \\
\text { the lack of conduction block } \\
\text { identified on comprehensive routine } \\
\text { neurophysiological testing? }\end{array}$ \\
\hline Pathophysiology & $\begin{array}{l}\text { Is MMN an axonal or demyelinating } \\
\text { neuropathy? } \\
\text { Is MMN a nodo-paranodopathy? } \\
\text { Are anti-GM1 IgM antibodies } \\
\text { pathogenic or a bystander? } \\
\text { Are there other relevant antibodies } \\
\text { when anti-GM1 is negative? }\end{array}$ \\
\hline Treatment & $\begin{array}{l}\text { What are other treatment } \\
\text { options apart from intravenous } \\
\text { immunoglobulin? } \\
\text { What are current treatment } \\
\text { recommendations based on the } \\
\text { available evidence? }\end{array}$ \\
\hline
\end{tabular}

occurs abruptly and, at least in the earliest stages of the disease, motor conduction distal to the site of block may remain normal. ${ }^{3}$

The criteria for definite $\mathrm{CB}$ have been debated at length, with different criteria for different nerves depending on difficulty of obtaining consistent compound motor action potentials (CMAPs) from appropriate sites. ${ }^{23-10}$ For example defining CB as definite in the tibial nerve requires a greater CMAP decrement than in the forearm segment of the median nerve. There is evidence that CMAP decrement for the detection of block may be more accurately measured from CMAP area decrement, rather than amplitude. ${ }^{9}$

Box 1 Clinical criteria for multifocal motor neuropathy as proposed by the EuropeanFederation of Neurological Societies $^{7}$

Core criteria-both must be present

- Slowly progressive or stepwise progressive, focal, asymmetrical limb weakness, that is, motor involvement in the motor nerve distribution of at least two nerves, for more than 1 month. If symptoms and signs are present in the distribution of one nerve only a possible diagnosis can be made

- No objective sensory abnormalities except for minor vibration sense abnormalities in the lower limbs

Supportive clinical criteria

- Predominant upper limb involvement

- Decreased or absent tendon reflexes in the affected limb

- Absence of cranial nerve involvement

- Cramps and fasciculations in the affected limb

- Response in terms of disability or muscle strength to immunomodulatory treatment

Exclusion criteria

- Upper motor neuron signs

- Marked bulbar involvement

- Sensory impairment more marked than minor vibration loss in the lower limbs

- Diffuse symmetrical weakness during the initial weeks

Copyright 2010 Peripheral Nerve Society. Used with permission from, John Wiley and Sons. ${ }^{7}$
Box 2 Electrophysiological criteria for conduction block $^{7}$

1. Definite motor conduction block $(\mathrm{CB})^{*}$

Negative peak compound motor action potential (CMAP) area reduction on proximal vs distal stimulation of at least $50 \%$ whatever the nerve segment length (median, ulnar and peroneal). Negative peak CMAP amplitude on stimulation of the distal part of the segment with motor CB must be $>20 \%$ of the lower limit of normal and $>1 \mathrm{mV}$ and increase of proximal to distal negative peak CMAP duration must be $\leq 30 \%$

2. Probable motor $C B^{*}$

Negative peak CMAP area reduction of at least $30 \%$ over a long segment of an upper limb nerve with increase of proximal to distal negative peak CMAP duration $\leq 30 \%$

OR

Negative peak CMAP area reduction of at least $50 \%$ (same as definite) with an increase of proximal to distal negative peak CMAP duration $>30 \%$

3. Normal sensory nerve conduction in upper limb segments with CB (see exclusion criteria)

Copyright 2010 Peripheral Nerve Society. Used with permission from John Wiley and Sons. ${ }^{7}$

*Evidence for CB must be found at sites distinct from common entrapment or compression syndromes.

Phase cancellation due to temporal dispersion leading to spurious $\mathrm{CB}$ is a common finding in demyelinating neuropathies and can be a significant issue in the diagnosis of $\mathrm{CB}$ in $\mathrm{MMN}{ }^{9}$ Currently there are no reliable and reproducible techniques for assessing proximal sites of CB. Needle stimulation of motor roots is difficult to undertake with significant chance of falsepositive recordings and is not well tolerated. Many criteria for $\mathrm{CB}$ in $\mathrm{MMN}$ also include a stipulation of the maximal amount of temporal dispersion that is allowable. Similarly, CB is very difficult to delineate reliably when the distal evoked CMAP amplitude is less than $1 \mathrm{mV}^{3}$

Electromyography (EMG) almost always reveals significant chronic denervation and reinnervation of muscles supplied by nerves with $\mathrm{CB}$, demonstrating that axonal degeneration is a significant feature of MMN even from the earliest onset of the disease. $^{3}$

There have been reports of MMN with typical clinical features but no $\mathrm{CB}$ identified. ${ }^{4} \mathrm{~A}$ possible reason is very proximal or distal locations of $\mathrm{CB}$ where routine electrophysiology is unable to detect block. Another possibility is conduction studies were performed only in clinically affected limbs while CB may be found also in nerves innervating muscles with normal strength. There does not appear to be any significant differences in clinical characteristics and treatment response between those with and without focal block. ${ }^{4}$

\section{Differential diagnosis}

In a patient who presents with symptoms of weakness suggestive of a lower motor neuron (LMN) syndrome, apart from $\mathrm{MMN}$, other diagnoses to consider include amyotrophic lateral sclerosis (ALS) and particularly the LMN variant of progressive muscular atrophy (PMA), late onset forms of spinal muscular atrophy (SMA), monomelic amyotrophy including Hirayama disease, focal and pure motor variants of chronic inflammatory demyelinating polyneuropathy (CIDP), multifocal acquired demyelinating sensory and motor neuropathy (MADSAM) often referred to as the Lewis-Sumner Syndrome, distal hereditary 
motor neuropathies and hereditary neuropathy with liability to pressure palsy (HNPP). ${ }^{11}$ The acute motor axonal neuropathy (AMAN) variant of Guillain-Barré syndrome (GBS) is a consideration in pure motor presentations with an acute onset. There have been reported cases of MMN presenting abruptly though this is unusual. ${ }^{12}$ Monitoring often reveals progression in MMN beyond 1 month, whereas AMAN tends to exhibit a monophasic course with plateau by 4 weeks from symptom onset followed by a period of recovery.

MMN is an important diagnosis to consider given there is at least partially effective treatment available. Some features are atypical for MMN, particularly the presence of bulbar or respiratory involvement, or sensory involvement. Differential diagnoses can generally be differentiated from MMN by appropriate imaging of the cervical spinal cord (Hirayama disease), electrophysiological (MADSAM, PMA), genetical (SMA, HNPP and a proportion of $\mathrm{dHMN}$ ), serological (AMAN typically associated with IgG antibodies against GM1 and GD1a) and in some cases cerebrospinal fluid analysis (MADSAM, CIDP and variants, GBS). ${ }^{11}$ Although in some cases the diagnosis is still difficult and serial assessment and even trials of therapy with appropriate monitoring are required.

\section{MRI and neuromuscular ultrasound}

A supportive criterion for the diagnosis of $\mathrm{MMN}$ is the detection of diffuse nerve swelling of the brachial plexus by MRI. ${ }^{7}$ Nerve ultrasound is also emerging as a tool to identify such abnormalities and possible sites of proximal CB.

MRI of the cervical nerve roots and brachial plexus can demonstrate abnormalities of cervical root and plexus T2 hyperintensity and enlargement in MMN in between 35\% to 50\% of patients. ${ }^{13} 14$ Such abnormalities can also be seen in other inflammatory neuropathies such as CIDP. An abnormal MRI did not correlate with specific clinical characteristics or response to treatment in MMN patients. ${ }^{13}$

High-resolution ultrasound (HRUS) is an emerging tool in the assessment of peripheral nerve pathology. Multifocal nerve enlargement can be identified in the brachial plexus, nerve roots and peripheral nerves, with these changes demonstrated in nerves both with and without clinical and electrophysiological abnormalities. ${ }^{15}$ One study found significantly increased cross-sectional areas (CSA) in median, ulnar and tibial nerves in MMN patients compared with controls. ${ }^{16}$ Intra-nerve variability and asymmetry of CSA was seen in the median nerve of MMN patients, suggestive of significant focality of nerve abnormalities.

A comparative study of MRI and HRUS of the cervical nerve roots and brachial plexus in treatment-naïve patients with CIDP or MMN demonstrated MRI findings of nerve enlargement and/or T2 hyperintensity in 50\% of MMN patients and HRUS finding of nerve enlargement in $67 \% .{ }^{14}$ Imaging abnormalities on either modality were seen in $79 \%$ of MMN patients, half of this group having both abnormal MRI and HRUS, 36\% with only HRUS abnormalities and 14\% with only MRI findings.

Ultrasound studies have shown promise in assisting with differentiating MMN from ALS. Studies of motor-containing peripheral nerves, cervical nerve roots and/or brachial plexus have found increased CSA measurements in MMN compared with ALS patients. ${ }^{17-19}$ Ultrasound protocols and models were also proposed to differentiate MMN and ALS, with sensitivities greater than $87 \%$ and specificities approaching $100 \%$ being shown. Apart from peripheral nerve CSA, a recent study using nerve ultrasound showed that median and ulnar nerve CSA distal-proximal ratios may be useful in differentiating ALS from
MMN. ${ }^{20}$ However it is unclear whether HRUS can distinguish between MMN and motor variants of CIDP.

Imaging abnormalities in $\mathrm{MMN}$ are generally multifocal and may correlate with sites of CB but can also be identified in nerves that do not have clinical or electrophysiological abnormalities detected. ${ }^{15} 171821$ This suggests that pathology may be more widespread than is evident by clinical and electrophysiological assessment alone, with imaging abnormalities potentially detectable at an earlier stage of disease course. Furthermore, diagnostic protocols consisting of examination at specific sites can be useful as shown in HRUS-based studies. ${ }^{18} 19$ HRUS-based assessment is likely to be a cheaper, safer and more accessible technique than MRI. However, both techniques require expertise at operation and interpretation and require population-based normal values to be defined for them to be part of an objective assessment.

\section{PATHOPHYSIOLOGY}

MMN is thought to be an immune-mediated neuropathy. The pathophysiological mechanism behind CB was initially hypothesised as due to focal demyelination, but there is increasing evidence that functional disturbances at the node of Ranvier may be the cause of block. The term 'nodo-paranodopathy' encompasses neuropathies due to dysfunction at nodes and paranodes. ${ }^{22}$ Schwann cell dysfunction at the paranodal region may be an important component of nodo-paranodopathy with very focal demyelination in this region necessary to open up the paranode to immune attack.

Nerve excitability studies of MMN showed excitability parameters consistent with hyperpolarisation, as also seen in postischaemic nerves, including greater axonal superexcitability distal to the site of $\mathrm{CB} .{ }^{23}$ Depolarisation at the site of $\mathrm{CB}$ may result in this observation via intracellular sodium $\left(\mathrm{Na}^{+}\right)$accumulation possibly due to sodium channel or $\mathrm{Na}^{+} /$potassium $\left(\mathrm{K}^{+}\right)$pump dysfunction. Recent mathematical modelling by Garg and colleagues suggested reductions of $\mathrm{Na}^{+}$and $\mathrm{K}^{+}$ion channel function along the axon distal to $\mathrm{CB}{ }^{24}$ These functional disturbances of the axonal membrane have been hypothesised to lead to increased ectopic generation manifesting as positive symptomatology such as fasciculations and cramps. ${ }^{23}$ A further study in which polarising direct currents were delivered at sites of $\mathrm{CB}$, as determined by inching studies, showed that blocks may be depolarising, hyperpolarising or both. ${ }^{25}$ Assessing axonal excitability at the sites of $\mathrm{CB}$ was based on response to the polarising current applied, with hyperpolarisation expected to worsen and depolarisation to improve $\mathrm{CB}$ at sites of membrane hyperpolarisation, with the opposite applying for sites of depolarisation. The authors hypothesised that their findings may reflect disease evolution over time with depolarising preceding the later development of hyperpolarising blocks.

$\mathrm{CB}$ in MMN has been demonstrated to be activity-dependent. One study showed transient reduction in proximal-to-distal CMAP ratios following maximal voluntary contraction (MVC). ${ }^{26}$ There was also reduction in force generated by affected muscles post-contraction. Transient CB or temporal dispersion was seen post-MVC in six MMN patients without overt $\mathrm{CB}$ identified on routine electrodiagnostic tests. ${ }^{27}$ Another study of 19 MMN patients failed to demonstrate activity-dependent CB. ${ }^{28}$ However in nerve segments with pre-existing $\mathrm{CB}$, MVC resulted in temporal dispersion. Apart from differences in methodology, the different findings between studies may reflect the nature or degree of the underlying block. The mechanism for these changes is likely nodal sodium channel dysfunction, $\mathrm{Na}^{+} / \mathrm{K}^{+}$-pump hyperactivity or other nodal/paranodal functional disturbance. 
Eighty-three per cent of MMN patients report cold paresis. ${ }^{5}$ This suggests a prominent functional disturbance rather than focal demyelination, as cold tends to improve symptoms and CB secondary to demyelination. Cooling of human nerves has been shown to induce axonal depolarisation, likely due to reduced activity of temperature-sensitive $\mathrm{Na}^{+} / \mathrm{K}^{+}$-pump. ${ }^{29}$ In $\mathrm{MMN}$, depolarising CBs may be exacerbated via this mechanism with corresponding symptom exacerbation. A study comparing the effect of cooling on excitability parameters in motor and sensory axons found significant differences between them. ${ }^{30}$ In motor axons, cooling resulted in changes consistent with axonal depolarisation, including fanning-in of threshold electrotonus (as defined by a decrease in threshold change of the threshold electrotonus waveforms) and steepening of current-voltage relation (I/V) slopes. ${ }^{30} 31$ These findings may reflect differences in ion channel subtypes expressed on motor and sensory axons, with their involvements in MMN possibly explaining its pure motor manifestations. Nerve excitability studies and the phenomenon of cold paresis indicate that MMN cannot be explained by demyelination alone, thereby supporting the concept of it being a nodo-paranodopathy.

\section{Anti-ganglioside antibodies}

The presence of anti-GM1 IgM antibodies was first described in $\mathrm{MMN}$ in 1988. These antibodies are present in at least $40 \%$ of cases. ${ }^{13} 32$ Sensitivity is low and they are detectable in a proportion of neurologically normal, ALS and other neuropathy patients. However in the setting of high positive titres and when an immune-mediated motor neuropathy is suspected, specificity can exceed $90 \% .{ }^{32}$ In MMN, these antibodies are likely produced by a limited number of B cell clones. ${ }^{33}$

The factors leading to development of MMN are not well understood. A genetic predisposition to autoimmunity is suggested by an increased frequency of other autoimmune diseases present in MMN patients and their first-degree relatives as well as higher frequencies of the human leucocyte antigen-DRB $1 * 15$ haplotype, which is also associated with multiple sclerosis and CIDP, among MMN patients relative to controls. ${ }^{34} 35$ A preceding infectious trigger may play a role, potentially through molecular mimicry of ganglioside or other epitopes, though there is not yet any clear evidence supporting this. A small study found antibodies against Campylobacter jejuni were more frequent than expected in MMN patients, but this was not supported by a later study. ${ }^{36}{ }^{37}$ IgM monoclonal gammopathy has been identified in $7 \%$ of MMN patients compared with $2 \%$ of healthy controls. ${ }^{38}$ The significance of this finding is not clear and may represent B cell autoimmunity or a concomitant lymphoproliferative disorder which may or may not be pathogenetically connected.

Gangliosides are glycosphingolipids attached to sialic acid moieties. GM1 is ubiquitously abundant and found in the central and peripheral nervous system. ${ }^{39}$ In peripheral nerves, it is found in the axolemma and myelin predominantly at nodal and paranodal regions and concentrated at cholesterol-enriched domains of plasma membranes. Gangliosides serve several functions including maintaining tight junctions, ion channel clustering with maintenance of potassium channels in paranodal/ internodal regions and sodium channels at nodes, and cellular calcium homeostasis. ${ }^{39-41}$

Studies have suggested that anti-GM1 antibodies are pathogenic through both direct and indirect mechanisms (figure 1). Anti-GM1 antibodies can cause direct functional disturbance via increase in potassium current at paranodes and disruption of calcium-signalling pathways. ${ }^{41} 42$ They can also cause indirect
A

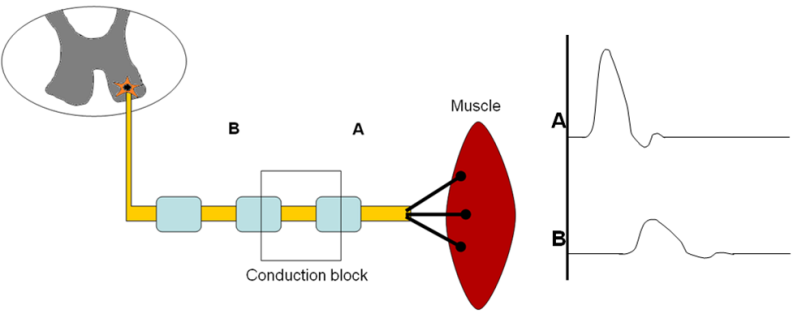

$B$

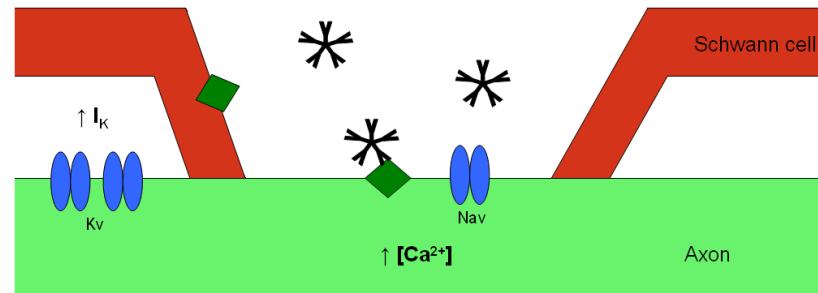

C
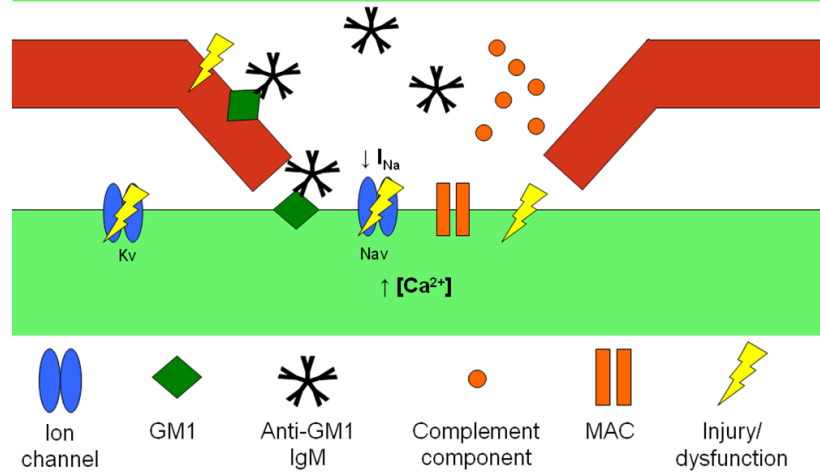

Figure 1 Simplified schematic diagram of a motor nerve myelinated axon nodal/paranodal region and potential mechanisms of nerve dysfunction and injury in multifocal motor neuropathy. Anti-GM1 IgM can cause both direct and indirect (via complement) disturbances of motor neurons and therefore result in focal conduction block (A). Direct effects include disruption of calcium homeostasis with an increase in intracellular calcium concentrations and an increase in potassium current (B). Indirect effects can be mediated through the classical complement pathway with membrane attack complex (MAC) formation (C). These can result in disruption of nodal sodium channel clusters, disruption of paranodes with altered potassium channel clusters and neuronal injury with ultimately axonal degeneration. NaV, voltage-gated sodium channel; Kv, voltagegated potassium channel; $I_{\mathrm{Na}^{\prime}}$ sodium current; $\mathrm{I}_{\mathrm{K}^{\prime}}$ potassium current.

nerve dysfunction via the classical complement pathway. ${ }^{43}$ In animal studies, complement activation and membrane attack complex formation result in disruption of nodal sodium channel clusters and paranodal potassium channel clusters. ${ }^{42}{ }^{44}$ These alterations can lead to suppression of sodium current and leakage of driving current. Complement deposition and activation was prevented by addition of immunoglobulins. ${ }^{43}$ A human induced-pluripotent stem cell (IPSC)-derived model of MMN showed both antibody-mediated complement-dependent and complement-independent disruption of motor neuron calcium homeostasis and axonal damage. ${ }^{41}$ Disruptions were reduced with immunoglobulin application. Though providing novel insights, the findings of this human in vitro model of $\mathrm{MMN}$ must be interpreted with its limitations in mind, which include the absence of glial cells and in particular Schwann cells and the relative immaturity of neurons studied.

Using indirect immunofluorescence on rat teased nerve fibres incubated with sera of $11 \mathrm{MMN}$ patients, Garg et al identified only one patient with a paranodal staining pattern despite $55 \%$ of patients being anti-GM1 IgM positive. ${ }^{24}$ In contrast, 9 of 11 
CIDP patients with $\mathrm{CB}$ showed staining at the node, paranode and/or myelin. This supports the notion that MMN and CIDP have differing disease mechanisms and target antigens. Though not supportive, this study does not rule out MMN as a possible nodo-paranodopathy. Differences between the human and rat epitope may limit antibody binding, and intravenous immunoglobulin (IVIG) given as treatment may also influence findings.

The focality of abnormalities raises questions as to the pathophysiological mechanisms resulting in such selectivity. Serum from patients with MMN has been shown to disrupt the bloodnerve barrier, ${ }^{45}$ with motor nerves more susceptible than sensory nerves. There is no significant difference in quantity of GM1 found on motor or sensory nerves, but differences in composition of ceramide moieties of gangliosides may modulate antibody binding affinity and susceptibility to antibody-mediated damage of motor and sensory nerves. ${ }^{46}$ GM1 serves a crucial role in maintaining the integrity of motor nerves but not as significantly for sensory nerves. ${ }^{40}$ Incubation of human IPSCderived sensory neurons with $\mathrm{MMN}$ patient sera resulted in binding of anti-GM1 antibodies to sensory neurites. ${ }^{41}$ However, the sensory neurites do not undergo damage as seen in motor neurites. This suggests that motor neurons are more vulnerable to anti-ganglioside antibody-mediated injury than sensory neurons. A possible explanation is the presence of cofactors on motor neurons, but absent on sensory neurons, playing a critical role in unmasking epitopes and facilitating nodal dysfunction and nerve injury. ${ }^{41}$

\section{Other antibodies and target antigens}

In a human IPSC-derived model, Harschnitz et al found that there was significantly increased $\operatorname{IgM}$ binding to neurites following incubation with either anti-GM1 IgM positive or negative MMN patient sera compared with controls. ${ }^{41}$ The antibody target of both sera groups appeared to be GM1. This finding is likely in part due to technical factors which limit sensitivity of anti-GM1 antibody detection. Several studies have shown increased detection rates of anti-GM1 IgM in combination with the glycolipid galactocerebroside (GalC), with increased sensitivity up to $81 \%$ but with a slight drop in specificity. ${ }^{47} 48$ The addition of GalC likely enhances antibody binding possibly through better exposure of GM1 epitopes.

Another possibility in anti-GM1 antibody-negative cases is the presence of antibodies directed at other antigens. Several studies have sought to identify these. The nodal proteins gliomedin and neurofascin-186 (NF186) have been proposed as potential targets given their role in nodal sodium channel clustering. Notturno et al showed $62 \%$ of MMN patients had antibodies to gliomedin or NF186 using synthetic rat peptides. However this was not supported by cell binding assays using transfected cells. ${ }^{49}$ Two studies which additionally examined for antibodies against paranodal proteins contactin-1 and neurofascin-155 using human proteins did not detect antibodies against these in any MMN patient. ${ }^{5051}$ The likely reason for these opposing results is the use of different proteins in their assays, rat in the first and human in the subsequent two studies. Pestronk et al proposed antibodies against heparin disaccharide NS6S as associated with acquired chronic motor neuropathies. ${ }^{52}$ They were present in $43 \%$ of patients with a motor neuropathy and $57 \%$ with MMN. Nobile-Orazio et al identified 23\% of MMN patients with antiNS6S IgM antibodies but this was not statistically significant compared with controls. ${ }^{47}$ Therefore anti-NS6S antibodies' diagnostic and pathogenic significance remains uncertain.

\section{Pathological changes}

There have been few studies examining the pathological changes of affected nerves. Kaji and colleagues described a patient who had their medial pectoral nerve removed for pathological examination, with this being just distal to the site of $\mathrm{CB}$ in the brachial plexus. $^{21}$ Demyelinating features of large-diameter axons that were thinly myelinated or devoid of myelin and small onion bulbs were seen with reduced fibre density. Inflammatory cell infiltrates were not seen. Auer et al reported a case of a lower motor neuron syndrome suggestive of MMN with electrophysiological findings consistent with demyelination in the brachial plexuses but with no CB identified. ${ }^{53}$ Proximal right ulnar nerve biopsy demonstrated features consistent with chronic demyelination with onion bulbs and an absence of inflammatory cells.

In contrast, Taylor and colleagues identified multifocal fibre degeneration and loss (particularly of large fibres) and significant numbers of regenerative clusters in fascicular nerve biopsies taken at sites of $\mathrm{CB}$ in eight nerves from seven $\mathrm{MMN}$ patients. ${ }^{54}$ There were no features to suggest demyelination including no onion-bulb formation. Small perivascular lymphocytic infiltrates were seen in two nerves. The authors hypothesised that MMN was an axonopathy without demyelination and that the CB was an antibody-mediated channelopathy focused at the nodes of Ranvier.

These two disparate findings, one of chronic demyelination and the other of multifocal fibre degeneration and regeneration, raise several possibilities. One possibility is that different pathological alterations occur at different sites of affected nerves, with chronic demyelinating changes at sites distal to CB perhaps secondary to the primary insult causing CB. Another possibility is that different underlying pathological mechanisms result in different pathological changes. Focal and motor variants of CIDP can have a clinical presentation very similar to that of MMN and also demonstrate motor CB on electrophysiological studies. However, nerve pathology in focal motor CIDP would be expected to demonstrate demyelination as its prominent feature (figure 2).

In terms of sensory nerve pathology, a study examining sensory nerve biopsies from 11 patients found very minor demyelinating features. ${ }^{55}$ These findings suggest that sensory nerves may also undergo insult though of much lesser severity compared with motor nerves. However, given the lack of sensory symptoms and electrophysiological abnormalities in $\mathrm{MMN}$, and in the cases biopsied, and these very minor pathological changes seen, it is difficult to make any strong inferences although sensory involvement in late stage disease has been reported in a significant number of cases. ${ }^{13}$

\section{TREATMENT}

Given its likely immunopathogenic basis, immunomodulating agents have been used extensively in the treatment of MMN. IVIG is currently the first-line treatment with evidence from several randomised controlled trials (RCT) demonstrating benefit. RCT evidence supporting non-immunoglobulin agents are however lacking.

\section{Intravenous immunoglobulin}

A beneficial effect of IVIG in improving muscle strength was first described in 1992. To date there have been five RCTs of IVIG use in MMN, all demonstrating a clear benefit. Four were part of a Cochrane meta-analysis which demonstrated an improvement in muscle strength in $78 \%$ of patients treated with IVIG compared with only $4 \%$ on placebo. ${ }^{56}$ Thirty-nine per cent of 

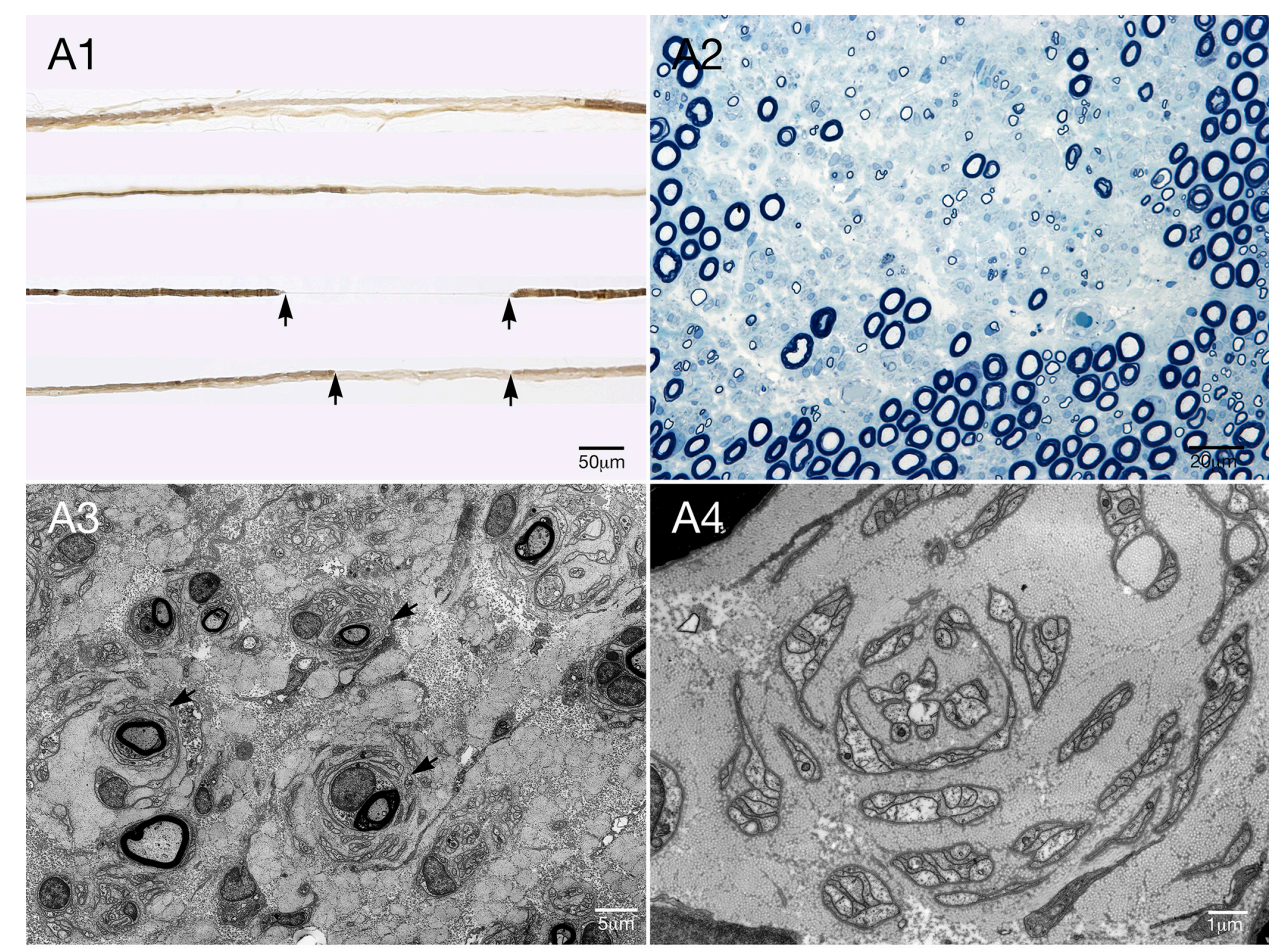

B1

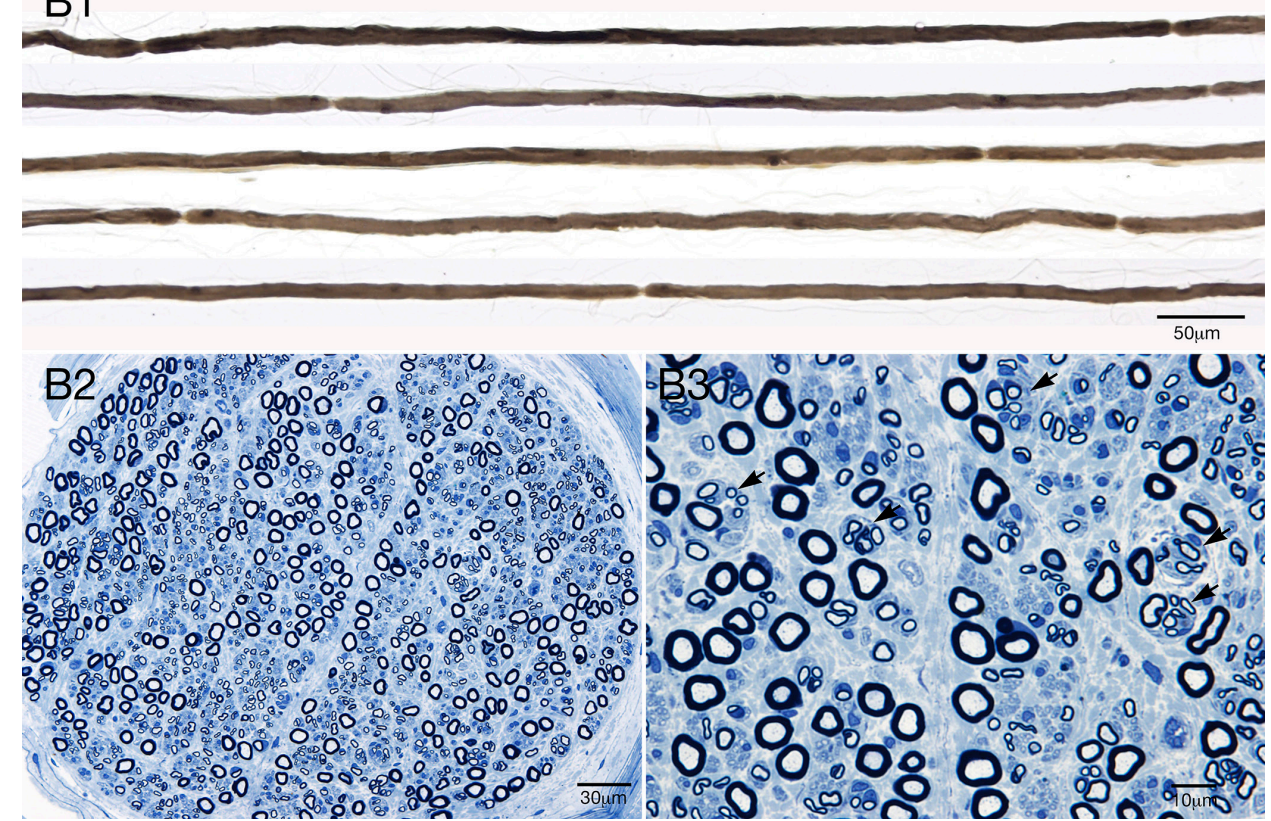

Figure 2 Proximal fascicular nerve biopsies taken from mixed motor and sensory nerves. Both cases had focal motor predominant neuropathies with some imaging abnormalities that allowed selection of nerve biopsy site. Case A (A1-A4) had focal hypertrophic demyelination and so was diagnosed with focal chronic inflammatory demyelinatingpolyneuropathy whereas case B (B1-B3) had mild neurogenic changes without evidence of inflammatory demyelination. Fascicular nerve biopsy from the left brachial plexus (a). (A1) Teased fibre preparations showing regions of demyelination between arrows. (A2) Semithin epoxy section stained with methylene blue shows apparent focal loss of myelinated fibres. (A3) Electron microscopy from region of fibre loss showing frequent small onion-bulb formations (arrows). (A4) Electron microscopy at a higher power showing a small onion-bulb with no fibre at the centre. Fascicular nerve biopsy from left median nerve at site of conduction block (B). (B1) Teased fibre preparations showing normal myelinated fibres without demyelination. (B2) Semithin epoxy section stained with methylene blue shows normal myelinated fibre density. (B3) On higher power, arrows showing regenerating cluster (arrows) and no onion-bulbs. (Courtesy of P. James B. Dyck and JaNean K. Engelstad).

IVIG-treated patients had improvement in disability but this was not statistically significant, which is likely due to lack of a good disability scale suitable for the unusual distribution of weakness in MMN. The final RCT was a double-blind crossover study with 44 patients randomised to two groups, one receiving 12 weeks of IVIG followed by 12 weeks of placebo and the other receiving the same treatments in reverse order. ${ }^{57}$ Mean maximal grip strength of the affected hand increased by $3.75 \%$ with IVIG and decreased $31.38 \%$ with placebo. Worsening disability was seen in $35.7 \%$ of subjects on placebo but not after crossing over to IVIG. This was compared with $11.9 \%$ whose disability worsened while on IVIG but not during their placebo period. 
Sixty-nine per cent of subjects were switched prematurely from placebo to open-label IVIG due to substantial functional deterioration on placebo. The authors concluded that IVIG treatment in MMN clearly improved muscle strength and disability.

In the long-term, most patients require maintenance IVIG to prevent clinical worsening. Despite continued IVIG, a significant proportion of patients exhibit disease progression with reduced muscle strength though still better than before treatment. ${ }^{1858-60}$ The IVIG dose required for maintenance increases with time. Serial electrophysiological assessment have demonstrated evidence of reinnervation and demyelination/axonal loss although CB may persist despite clinical improvement. ${ }^{60}$ IVIG, though beneficial, is only able to partially control the disease process and slow axon loss.

Cats et al showed that more severe disability or weakness was associated with axon loss and years untreated. ${ }^{1}$ Axon loss was the most significant determinant of muscle weakness followed by years untreated in another study. ${ }^{61}$ A study using higher maintenance doses of IVIG showed that IVIG can significantly reduce axonal degeneration and also promote reinnervation. ${ }^{62}$ As such, early diagnosis and treatment institution is of utmost importance to minimise axonal loss and disability accrual.

\section{Subcutaneous immunoglobulin}

Subcutaneousimmunoglobulin (SCIG) has potential advantages over IVIG including patient convenience, avoidance of hospitalisation, potential cost benefit and better overall adverse effect profile. SCIG also results in a more stable serum IgG level thus preventing adverse events due to unphysiologically high serum IgG levels and symptom fluctuation due to end-of-dose effect with IVIG.

Several studies have described SCIG therapy in patients who have previously been on maintenance IVIG, using equivalent total monthly doses. These have shown similar efficacy between SCIG and IVIG. ${ }^{63}$ A meta-analysis examining efficacy and safety of SCIG compared with IVIG in MMN and CIDP showed no significant differences in muscle strength and a $28 \%$ reduction in relative risk of moderate or systemic adverse effects. ${ }^{64} \mathrm{~A} 2$ year follow-up study showed SCIG was effective at maintaining clinical stability. ${ }^{65}$

An open-label trial of SCIG with 15 MMN patients used a dose ratio of 1.53:1 of SCIG-to-IVIG in patients receiving less than $2 \mathrm{~g} / \mathrm{kg}$ IVIG per month, and 1:1 dosing in patients on $2 \mathrm{~g} /$ $\mathrm{kg} /$ month. ${ }^{66}$ Three of six patients receiving equivalent dose SCIG deteriorated in strength while all patients on 1.53:1 SCIG dosing remained stable. The authors proposed that SCIG dosing should be commenced at a ratio greater than $1: 1$. This contrasts with studies demonstrating stability with equivalent dosing.

\section{Cyclophosphamide}

There are reports from the 1990's that have shown clinical benefit with use of oral or intravenous cyclophosphamide including in cases refractory to IVIG. ${ }^{67}$ However there are also descriptions of cases in which the efficacy of cyclophosphamide is equivocal or not effective at all. ${ }^{59}$

In a study of six MMN patients on maintenance IVIG, oral cyclophosphamide was added. ${ }^{67}$ All six patients showed improvement in their muscle strength, functional impairment scores and modified Rankin disability scores. They were able to increase the interval between IVIG doses, with three patients able to stop all treatment for up to 2 years before return of symptoms which responded to IVIG. Longer follow-up of these patients has not been reported
Cyclophosphamide has potentially significant adverse effects including myelosuppression, haemorrhagic cystitis, infertility, malignancies and opportunistic infections. ${ }^{67}$ Risk increases with repeated treatment which is usually necessary in MMN. In a patient who is refractory to IVIG with significant worsening of weakness and/or functional impact, treatment with cyclophosphamide may be trialled but must be considered in the context of its risk profile. Full informed consent and careful monitoring are essential. It is important to note that there is little evidence that cyclophosphamide or any therapy will reverse established disability and therefore the use of potentially harmful therapies should be avoided in this situation. Given mixed reports of benefit, an RCT would be very useful in clarifying cyclophosphamide's efficacy, and if this is established also the ideal delivery route and dosage regimen. However, the development and application of newer therapies has made such a trial highly unlikely. Therefore, cyclophosphamide as a treatment for MMN should be considered a therapy of last resort with full informed consent in those who are progressing despite best practice treatment with IVIG and whose independence is significantly threatened by progressive MMN.

\section{Steroids and plasma exchange}

Corticosteroid efficacy in $\mathrm{MMN}$ has been disappointing with no benefit and in some cases clinical worsening. ${ }^{59}$ A similar situation has been observed with plasma exchange with a lack of efficacy and again with some patients exhibiting deterioration. ${ }^{68}$ The reason for these unexpected responses to steroids and plasma exchange is unknown, but may be due to an altered balance between damaging and regulatory immune components resulting in worsening clinical state.

\section{Rituximab}

Rituximab is a murine-human chimeric monoclonal antibody against CD20, which results in B cell depletion. Several small observational studies described benefit with rituximab, with improved muscle strength and increased interval between IVIG doses in those on maintenance IVIG. ${ }^{69}$ Other studies reported equivocal benefit in terms of maintenance IVIG requirement, with one case describing a clinical worsening 2 months following rituximab dosing. ${ }^{70}$ An open-label trial of six patients on maintenance IVIG given rituximab and followed for 12 months did not demonstrate a reduction in IVIG administration or change in grip strength, Medical Research Council sum score or disability score. $^{71}$ Further studies of rituximab and other anti-CD20 monoclonal antibodies are required to determine if there is any benefit. Currently the use of rituximab in MMN cannot be supported outside of clinical trials or possibly as rescue therapy where other treatments have failed, with a low expectation of success.

\section{Eculizumab}

Eculizumab is a humanised monoclonal antibody against the complement component C5 which acts to inhibit the terminal pathway in complement activation. Given complement activation appears to be implicated in the pathophysiology of MMN, inhibition would be expected to prevent nerve injury. Eculizumab has been proven to be beneficial in paroxysmal nocturnal haemoglobinuria, a disease in which complement activation is involved in its pathogenesis. In an open-label trial of eculizumab in 13 MMN cases, improvements in self-evaluated functional rating scale and muscle strength as measured by myometry, as well as a small but statistically significant decrease in median percentage 
of $\mathrm{CB}$ across all nerves studied, were seen. ${ }^{72}$ No change in IVIG dosing interval in those on maintenance IVIG was seen. This short-term non-blinded trial demonstrates the potential clinical benefit with eculizumab, but this needs to be established in larger RCTs with longer follow-up.

\section{Other immunomodulatory treatments}

There are reports of other immunomodulatory agents being used, including azathioprine and interferon-beta, with variable effect. $^{8}{ }^{73}$ Adjunctive use of mycophenolate was assessed in a RCT with 28 patients. ${ }^{74}$ No significant alteration in disease course or IVIG dose was seen. Thus, there is no compelling evidence to advocate treatment with these agents at this stage.

\section{Treatment conclusions}

Currently the only treatment that has been shown to be unequivocally useful in the treatment of MMN is IVIG/SCIG. However, there is no clear consensus on treatment regimes and when to reduce, stop or commence therapy. Many cases of MMN may demonstrate stability for years without therapy and treatment may be seen as holding them stable. Therefore, understanding the trajectory of an individual's condition is important. Regular review with nerve conduction studies and a blinded assessment of peripheral nerve function such as the neuropathy impairment score (NIS) or NIS subscore of weakness can significantly aid in treatment decisions. ${ }^{375}$ No treatment has been shown to reverse established atrophy. A trajectory of worsening on assessment is an indication for treatment, and this should consist of IVIG or SCIG utilising the regimens as outlined in the clinical trials. If improvement or stabilisation of a worsening trajectory is seen the maintenance dosage of IVIG/SCIG that maintains that improvement should be sought. Practice points proposed by the European Federation of Neurological Societies and the Peripheral Nerve Society suggest IVIG induction dose of $2 \mathrm{~g} / \mathrm{kg}$ over 2 to 5 days, and maintenance IVIG of $1 \mathrm{~g} / \mathrm{kg}$ every 2 to 4 weeks or $2 \mathrm{~g} / \mathrm{kg}$ every 1 to 2 months as guided by treatment response. IVIG/SCIG is expensive and in short supply in many countries and therefore every effort should be made to limit the amount used to treat MMN as for all conditions. Clear cut guidelines for stopping or reducing therapy should be developed based on trials of weaning or ceasing therapy.

Other immunosuppresants/immunomodulators (cyclophosphamide, rituximab or eculizumab) have not been shown to alter the natural history of MMN reproducibly in clinical trials. Preferably these treatments should be given as part of clinical trials. However due to the rarity of MMN these are not currently underway to the best of our knowledge. Fully informed consent and careful objective monitoring is essential.

\section{CONCLUSION}

Since MMN was first described more than three decades ago, our understanding of its clinical characteristics and underlying pathophysiology have progressed. Early recognition and diagnosis of MMN is important given that the years left untreated and resultant axonal loss are poor prognostic factors. ${ }^{161}$ Brachial plexus MRI has been shown to be useful in diagnostic evaluation, and HRUS is also an emerging and promising technique. Testing for antibodies against GM1 with GalC in patients negative for anti-GM1 antibodies may aid evaluation. The aim once MMN is diagnosed is to institute effective treatment to slow disease progression and axonal loss. Currently IVIG/SCIG is the treatment of choice although there are still many unanswered treatment questions. Eculizumab has been proposed as a possible effective treatment, but we currently await RCT evidence regarding its efficacy.

The pathophysiology of MMN is still not fully elucidated however there is now strong evidence that MMN is not a variant of CIDP and that these two conditions can be separated pathologically. There is increasing evidence that MMN is a disorder of the paranodal or nodal regions of the node of Ranvier. However direct ultrastructural confirmation of this is lacking and should be a major goal of future research. The mechanisms by which an immune attack can result in focal motor nerve damage presumably acting at the nodes of Ranvier need further elucidation. The development of an IPSC model of MMN and other peripheral nerve disorders will greatly aid these types of experiments.

Contributors BVT, MCK, PJBD and LHvdB conceived the idea for the article. WZY drafted the manuscript. All authors revised the manuscript critically for important intellectual content and gave final approval of the version to be published.

Funding BVT was supported by a Macquarie Foundation MSRA Clinical Fellowship. This work was supported in part by funding to Forefront from the National Health and Medical Research Council of Australia (NHMRC) program grant (\#1037746). MCK was supported by an NHMRC Practitioner Fellowship (\#1156093).

Competing interests None declared.

Patient consent for publication Not required.

Provenance and peer review Not commissioned; externally peer reviewed.

ORCID iD

Wei Zhen Yeh http://orcid.org/0000-0002-5335-6612

\section{REFERENCES}

1 Cats EA, der PW-Lvan, Piepers $S$, et al. Correlates of outcome and response to IVIg in 88 patients with multifocal motor neuropathy. Neurology 2010;75:818-25.

2 Nobile-Orazio E. Multifocal motor neuropathy. J Neuroimmunol 2001;115:4-18.

3 Taylor BV, Wright RA, Harper CM, et al. Natural history of 46 patients with multifocal motor neuropathy with conduction block. Muscle Nerve 2000;23:900-8.

4 Slee M, Selvan A, Donaghy M. Multifocal motor neuropathy: the diagnostic spectrum and response to treatment. Neurology 2007;69:1680-7.

5 Straver DCG, van Asseldonk J-TH, Notermans NC, et al. Cold paresis in multifocal motor neuropathy. J Neurol 2011;258:212-7.

6 Galassi G, Albertini G, Valzania F, et al. Cranial nerve involvement as presenting sign of multifocal motor neuropathy. J Clin Neurosci 2012;19:1733-5.

7 Joint Task Force of the EFNS and the PNS. Report of a joint Task force of the European Federation of neurological societies and the peripheral nerve Society - first revision. $J$ Peripher Nerv Syst 2010;15:295-301.

8 Bouche P, Moulonguet A, Younes-Chennoufi AB, et al. Multifocal motor neuropathy with conduction block: a study of 24 patients. J Neurol Neurosurg Psychiatry 1995:59:38-44.

9 Rhee EK, England JD, Sumner AJ. A computer simulation of conduction block: effects produced by actual block versus interphase cancellation. Ann Neurol 1990;28:146-56

10 Van Asseldonk JTH, Van den Berg LH, Wieneke GH, et al. Criteria for conduction block based on computer simulation studies of nerve conduction with human data obtained in the forearm segment of the median nerve. Brain J Neuro/ 2006;129:2447-60.

11 Garg N, Park SB, Vucic S, et al. Differentiating lower motor neuron syndromes. J Neurol Neurosurg Psychiatry 2017;88:474-83.

12 Lefaucheur J, Gregson N, Gray I, et al. A variant of multifocal motor neuropathy with acute, generalised presentation and persistent conduction blocks. J Neurol Neurosurg Psychiatry 2003;74:1555-61.

13 Jongbloed BA, Bos JW, Rutgers D, et al. Brachial plexus magnetic resonance imaging differentiates between inflammatory neuropathies and does not predict disease course. Brain Behav 2017;7.

14 Goedee HS, Jongbloed BA, van Asseldonk J-TH, et al. A comparative study of brachial plexus sonography and magnetic resonance imaging in chronic inflammatory demyelinating neuropathy and multifocal motor neuropathy. Eur J Neurol 2017;24:1307-13.

15 Beekman $\mathrm{R}$, van den Berg LH, Franssen $\mathrm{H}$, et al. Ultrasonography shows extensive nerve enlargements in multifocal motor neuropathy. Neurology 2005:65:305-7.

16 Kerasnoudis A, Pitarokoili K, Behrendt V, et al. Multifocal motor neuropathy: correlation of nerve ultrasound, electrophysiological, and clinical findings. J Peripher Nerv Syst JPNS 2014;19:165-74

17 Grimm A, Décard BF, Athanasopoulou I, et al. Nerve ultrasound for differentiation between amyotrophic lateral sclerosis and multifocal motor neuropathy. $J$ Neurol 2015;262:870-80. 
18 Loewenbrück KF, Liesenberg J, Dittrich M, et al. Nerve ultrasound in the differentiation of multifocal motor neuropathy (MMN) and amyotrophic lateral sclerosis with predominant lower motor neuron disease (ALS/LMND). J Neurol 2016;263:35-44.

19 Goedee HS, van der Pol WL, van Asseldonk J-TH, et al. Diagnostic value of sonography in treatment-naive chronic inflammatory neuropathies. Neurology 2017;88:143-51.

20 Noto Y-I, Garg N, Li T, et al. Comparison of cross-sectional areas and distal-proximal nerve ratios in amyotrophic lateral sclerosis. Muscle Nerve 2018;58:777-83.

21 Kaji R, Oka N, Tsuji T, et al. Pathological findings at the site of conduction block in multifocal motor neuropathy. Ann Neurol 1993;33:152-8.

22 Uncini A, Susuki K, Yuki N. Nodo-paranodopathy: beyond the demyelinating and axonal classification in anti-ganglioside antibody-mediated neuropathies. Clin Neurophysiol Off J Int Fed Clin Neurophysiol 2013;124:1928-34.

23 Kiernan MC, Guglielmi J-M, Kaji R, et al. Evidence for axonal membrane hyperpolarization in multifocal motor neuropathy with conduction block. Brain 2002:125:664-75.

24 Garg N, Park SB, Howells J, et al. Conduction block in immune-mediated neuropathy: paranodopathy versus axonopathy. Eur J Neurol 2019;13953.

25 Priori A, Bossi B, Ardolino G, et al. Pathophysiological heterogeneity of conduction blocks in multifocal motor neuropathy. Brain 2005;128:1642-8.

26 Kaji R, Bostock H, Kohara N, et al. Activity-Dependent conduction block in multifocal motor neuropathy. Brain J Neurol 2000;123:1602-11.

27 Nodera H, Bostock H, Izumi Y, et al. Activity-Dependent conduction block in multifocal motor neuropathy: magnetic fatigue test. Neurology 2006;67:280-7.

28 Straver DCG, van den Berg LH, van den Berg-Vos RM, et al. Activity-Dependent conduction block in multifocal motor neuropathy. Muscle Nerve 2011;43:31-6.

29 Franssen H, Gebbink TA, Wokke JHJ, et al. Is cold paresis related to axonal depolarization? J Peripher Nerv Syst 2010;15:227-37.

30 Kovalchuk MO, Franssen $\mathrm{H}$, Van Schelven LJ, et al. Comparing excitability at $37^{\circ} \mathrm{C}$ versus at $20^{\circ} \mathrm{C}$ : differences between motor and sensory axons. Muscle Nerve (Published Online First: 6 September 2017).

31 Burke D, Kiernan MC, Bostock H. Excitability of human axons. Clin Neurophysiol 2001;112:1575-85.

32 Taylor BV, Gross L, Windebank AJ. The sensitivity and specificity of anti-GM1 antibody testing. Neurology 1996;47:951-5.

33 Cats EA, van der Pol W-L, Tio-Gillen AP, et al. Clonality of anti-GM1 IgM antibodies in multifocal motor neuropathy and the Guillain-Barré syndrome. J Neurol Neurosurg Psychiatry 2015;86:502-4.

34 Cats EA, Bertens AS, Veldink JH, et al. Associated autoimmune diseases in patients with multifocal motor neuropathy and their family members. J Neurol 2012;259:1137-41

35 Sutedja NA, Otten HG, Cats EA, et al. Increased frequency of HLA-DRB1 * 15 in patients with multifocal motor neuropathy. Neurology 2010;74:828-32.

36 Taylor BV, Phillips BA, Speed BR, et al. Serological evidence for infection with Campylobacter jejuni/coli in patients with multifocal motor neuropathy. $J$ Clin Neurosci 1998;5:33-5.

37 Terenghi F, Allaria S, Scarlato G, et al. Multifocal motor neuropathy and Campylobacter jejuni reactivity. Neurology 2002;59:282-4.

38 Vlam L, Piepers S, Sutedja NA, et al. Association of IgM monoclonal gammopathy with progressive muscular atrophy and multifocal motor neuropathy: a case-control study. J Neurol 2015;262:666-73.

39 Willison HJ, Yuki N. Peripheral neuropathies and anti-glycolipid antibodies. Brain J Neurol 2002;125:2591-625.

40 Susuki K, Baba H, Tohyama K, et al. Gangliosides contribute to stability of paranodal junctions and ion channel clusters in myelinated nerve fibers. Glia 2007;55:746-57.

41 Harschnitz O, van den Berg LH, Johansen LE, et al. Autoantibody pathogenicity in a multifocal motor neuropathy induced pluripotent stem cell-derived model. Ann Neurol 2016:80:71-88

42 Takigawa T, Yasuda H, Kikkawa R, et al. Antibodies against GM1 ganglioside affect $\mathrm{K}+$ and $\mathrm{Na}+$ currents in isolated rat myelinated nerve fibers. Ann Neurol 1995;37:436-42.

43 Yuki N, Watanabe H, Nakajima T, et al. Ivig blocks complement deposition mediated by anti-GM1 antibodies in multifocal motor neuropathy. J Neurol Neurosurg Psychiatry 2011;82:87-91.

44 Susuki K, Rasband MN, Tohyama K, et al. Anti-Gm1 antibodies cause complementmediated disruption of sodium channel clusters in peripheral motor nerve fibers. J Neurosci 2007:27:3956-67.

45 Shimizu F, Omoto M, Sano Y, et al. Sera from patients with multifocal motor neuropathy disrupt the blood-nerve barrier. J Neurol Neurosurg Psychiatry 2014;85:526-37.

46 Ogawa-Goto K, Abe T. Gangliosides and glycosphingolipids of peripheral nervous system Myelins-a minireview. Neurochem Res 1998;23:305-10.

47 Nobile-Orazio E, Giannotta C, Musset L, et al. Sensitivity and predictive value of anti-GM1/galactocerebroside IgM antibodies in multifocal motor neuropathy. J Neurol Neurosurg Psychiatry 2014;85:754-8.
48 Galban-Horcajo F, Vlam L, Delmont E, et al. The diagnostic utility of determining antiGM1: GALC complex antibodies in multifocal motor neuropathy: a validation study. J Neuromuscul Dis 2015;2:157-65.

49 Notturno F, Di Febo T, Yuki N, et al. Autoantibodies to neurofascin-186 and gliomedin in multifocal motor neuropathy. J Neuroimmunol 2014;276:207-12.

50 Doppler K, Appeltshauser L, Krämer HH, et al. Contactin-1 and Neurofascin-155/-186 are not targets of auto-antibodies in multifocal motor neuropathy. PLOS ONE 2015;10.

51 Mathey EK, Garg N, Park SB, et al. Autoantibody responses to nodal and paranodal antigens in chronic inflammatory neuropathies. J Neuroimmunol 2017;309:41-6.

52 Pestronk A, Chuquilin M, Choksi R. Motor neuropathies and serum IgM binding to NS6S heparin disaccharide or GM1 ganglioside. J Neurol Neurosurg Psychiatry 2010:81:726-30

53 Auer RN, Bell RB, Lee MA. Neuropathy with onion bulb formations and pure motor manifestations. Can J Neuro/ Sci J Can Sci Neurol 1989:16:194-7.

54 Taylor BV, Dyck PJB, Engelstad J, et al. Multifocal motor neuropathy: pathologic alterations at the site of conduction block. J Neuropathol Exp Neurol 2004;63:129-37

55 Corse AM, Chaudhry V, Crawford TO, et al. Sensory nerve pathology in multifocal motor neuropathy. Ann Neurol 1996;39:319-25.

56 van Schaik IN, van den Berg LH, de Haan R, et al. Intravenous immunoglobulin for multifocal motor neuropathy. Cochrane Database Syst Rev 2005:CD004429.

57 Hahn AF, Beydoun SR, Lawson V, et al. A controlled trial of intravenous immunoglobulin in multifocal motor neuropathy. J Peripher Nerv Syst JPNS 2013; 18:321-30

58 Léger J-M, Viala K, Cancalon F, et al. Intravenous immunoglobulin as short- and long-term therapy of multifocal motor neuropathy: a retrospective study of response to IVIg and of its predictive criteria in 40 patients. J Neurol Neurosurg Psychiatry 2008;79:93-6

59 Azulay JP, Rihet P, Pouget J, et al. Long term follow up of multifocal motor neuropathy with conduction block under treatment. J Neurol Neurosurg Psychiatry 1997;62:391-4.

60 Van den Berg-Vos RM, Franssen H, Wokke JHJ, et al. Multifocal motor neuropathy: long-term clinical and electrophysiological assessment of intravenous immunoglobulin maintenance treatment. Brain J Neurol 2002;125:1875-86.

61 Van Asseldonk JTH, Van den Berg LH, Kalmijn S, et al. Axon loss is an important determinant of weakness in multifocal motor neuropathy. J Neurol Neurosurg Psychiatry 2006;77:743-7

62 Vucic S, Black KR, Chong PST, et al. Multifocal motor neuropathy: decrease in conduction blocks and reinnervation with long-term IVIg. Neurology 2004;63:1264-9.

63 Harbo T, Andersen $\mathrm{H}$, Hess A, et al. Subcutaneous versus intravenous immunoglobulin in multifocal motor neuropathy: a randomized, single-blinded cross-over trial. Eur J Neurol 2009:16:631-8

64 Racosta JM, Sposato LA, Kimpinski K. Subcutaneous versus intravenous immunoglobulin for chronic autoimmune neuropathies: a meta-analysis. Muscle Nerve 2017; 55:802-9.

65 Harbo T, Andersen H, Jakobsen J. Long-Term therapy with high doses of subcutaneous immunoglobulin in multifocal motor neuropathy. Neurology 2010;75:1377-80.

66 Katzberg HD, Rasutis V, Bril V. Subcutaneous immunoglobulin for treatment of multifocal motor neuropathy. Muscle Nerve 2016;54:856-63.

67 Meucci N, Cappellari A, Barbieri S, et al. Long term effect of intravenous immunoglobulins and oral cyclophosphamide in multifocal motor neuropathy. $J$ Neurol Neurosurg Psychiatry 1997;63:765-9.

68 Claus D, Specht S, Zieschang M. Plasmapheresis in multifocal motor neuropathy: a case report. J Neurol Neurosurg Psychiatry 2000;68:533-5.

69 Pestronk A, Florence J, Miller T, et al. Treatment of IgM antibody associated polyneuropathies using rituximab. J Neurol Neurosurg Psychiatry 2003;74:485-9.

70 Gorson KC, Natarajan N, Ropper AH, et al. Rituximab treatment in patients with IVIg-dependent immune polyneuropathy: a prospective pilot trial. Muscle Nerve 2007:35:66-9.

71 Chaudhry V, Cornblath DR. An open-label trial of rituximab (Rituxan $®$ ) in multifocal motor neuropathy. J Peripher Nerv Syst JPNS 2010;15:196-201.

72 Fitzpatrick AM, Mann CA, Barry S, et al. An open label clinical trial of complement inhibition in multifocal motor neuropathy. J Peripher Nerv Syst JPNS 2011;16:84-91.

73 Martina I, van Doorn PA, Schmitz P, et al. Chronic motor neuropathies: response to interferon- $\beta 1$ a after failure of conventional therapies. J Neurol Neurosurg Psychiatry 1999:66:197-201.

74 Piepers S, Van den Berg-Vos R, Van der Pol W-L, et al. Mycophenolate mofetil as adjunctive therapy for MMN patients: a randomized, controlled trial. Brain J Neurol 2007; 130:2004-10.

75 Dyck PJ, Taylor BV, Davies JL, et al. Office immunotherapy in chronic inflammatory demyelinating polyneuropathy and multifocal motor neuropathy. Muscle Nerve 2015:52:488-97. 